\title{
Causality analysis of feedforward-controlled systems with broadband inputs
}

\author{
R. A. Burdisso, J. S. Vipperman, and C. R. Fuller \\ Vibration and Acoustics Laboratories, Mechanical Engineering Department, Virginia Polytechnic \\ Institute and State University, Blacksburg, Virginia 24061-0238
}

(Received 14 May 1992; revised 24 November 1992; accepted 23 February 1993)

\begin{abstract}
In recent years adaptive feedforward control algorithms have been successfully implemented to attenuate the response of systems under persistent disturbances such as single and multiple tones as well as random inputs. System causality is not an issue when the excitation is sinusoidal because of its deterministic nature. However, causality is a very important factor in broadband control. Though significant deterioration in the performance of noncausal control systems have been reported in the literature, analytical tools are virtually nonexistent to predict the behavior of broadband controllers. The main objective of this research is to develop an approach to investigate system causality. A formulation is presented to address the effectiveness of a control configuration as a function of the filter size, delay time, and dynamic properties of the structure. The technique is illustrated in a simple numerical example and the results are also corroborated experimentally.
\end{abstract}

PACS numbers: $43.40 . \mathrm{Vn}$

\section{INTRODUCTION}

Persistent vibrations in elastic structures and their radiated sound fields are problems commonly found in engineering practice. Adaptive feedforward control algorithms have proved to be successful in attenuating stationary disturbances such as single, multiple frequencies, and random inputs. Adaptive feedforward algorithms were first applied to signal noise canceling as demonstrated by Widrow. ${ }^{1}$ More recently these techniques have been extended to control one-dimensional, ${ }^{2}$ multidimensional acoustic fields, ${ }^{3}$ as well as structurally radiated sound using structural control inputs. ${ }^{4}$ Feedforward least-mean-square (LMS) and recursive-least-mean-square (RLMS) adaptive algorithms have been applied on active control of bending motion in infinite or semi-infinite thin beams. ${ }^{5-8}$ Recently, the simultaneous control of flexural and extensional waves in beams has been demonstrated by a multichannel LMS approach, in conjunction with specialized piezoceramic transducers. ${ }^{9}$

However, most previous theoretical and experimental studies consider only single- and multiple-sinusoidal excitations. Applications of feedforward control for broadband excitation is much more scarce and mostly restricted for attenuating noise in ducts ${ }^{10-12}$ and enclosures. ${ }^{13,14}$ Active feedforward control of broadband flexural waves in infinite beams have being reported by Elliott. ${ }^{15}$ Recently, the authors have developed two new broadband feedforward control configurations for finite structures, ${ }^{16}$ which use an adaptive finite impulse response (FIR) filter as a compensator.

A general arrangement of a feedforward control approach applied to a finite system is shown in Fig. 1. The response of the system produced by the offending disturbance $x(t)$ is canceled by a "coherent" control input $u(t)$, which is obtained by tapping the disturbance input and feeding this signal into the compensator $W$. The compen- sator is computed such that the mean square value of some system response, i.e., the signal from an error sensor, is minimized. The feedforward control system is said to be causal when the signal fed into the compensator is obtained far enough in advance to allow time for the processing and propagation of the control signal to meet and cancel the disturbance signal at the error sensor. In other words, the propagation time of the disturbance $x(t)$ through the control path should be less than or equal to the propagation time through the disturbance path. System causality is not a constraint when the excitation is sinusoidal because of the deterministic nature of the signal. Future values of the signal are completely predictable under steady-state conditions. On the other hand, causality is a very important issue in broadband control because future values of the excitation cannot be predicted due to the randomness of this type of excitation. The signal is only predictable in some statistical sense. In feedforward broadband control of infinitely long systems, such as ducts and beams where there are no reflected waves from boundaries, the response to the disturbance can always be sensed far enough upstream to yield a causal system. In finite reverberant systems, where standing wave phenomena occur at the natural frequencies, it could be difficult or virtually impossible to configure the control system to render the system causal.

Early broadband control experiments have shown deterioration in the performance due to delays in the control path that results in noncausal control systems. Warner and Bernhard ${ }^{13}$ discussed qualitatively the influence of control path delay on the cancellation of nondeterministic sound in acoustic enclosures, while Sutton and his colleagues ${ }^{14}$ have experimentally demonstrated significant performance reduction in the control of random sound in enclosures by delaying the signal in the control path. They suggested improvement can be gained by placing the reference sensor close to the disturbance source and the control speaker 


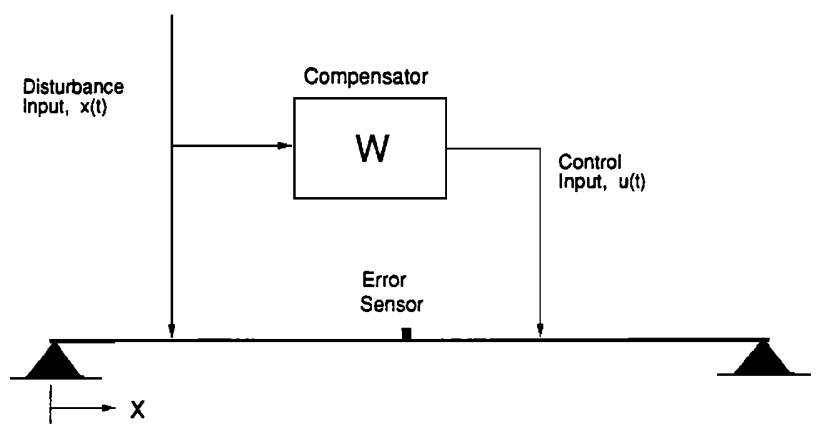

FIG. 1. General arrangement of a feedforward control approach.

close to the error or cancellation transducer. Similar performance degradation has also been observed in broadband structural vibration control experiments. ${ }^{16}$ Analytical studies on the performance limits of causally constrained control systems have also been reported in the literature. Nelson et al. ${ }^{17}$ have developed a time-domain formulation for the case of active control of sound. Joplin et al. ${ }^{18}$ adopted a numerical scheme to find the optimum causal filter in control of low frequency random sound in enclosures.

In practical implementation of digital controllers, the total control path delay is due to the smoothing filters, the sampling process for the controller, and the physical system itself. A causal controller can often be achieved by carefully choosing the distances between the disturbance and control inputs and the error sensors. This is particularly true in active control of acoustic enclosures where the wave speed is relatively low and the physical dimensions of the system are large. However, a causal system would be difficult to realize in many other situations, i.e., in active control of structural vibrations. Thus, the main goal of this work is to investigate the control performance of noncausal control systems, and to lay out the mathematical foundations to develop design guidelines of noncausal feedforward controllers. In contrast to the work of Nelson et al. ${ }^{17}$ the formulation presented here is carried out in the frequencydomain. The analysis demonstrates that some control is always achievable for any degree of "noncausality" in a system. The work also shows that the performance degradation due to delay in the control path can be offset by increasing the compensator filter size. The proposed formulation is then applied to the case of a simply supported beam for illustrative results and also compared to an experimental investigation of the same system.

\section{THEORY}

The single input, single output feedforward control system shown in Fig. 2 is considered. The analysis is first carried out in the discrete time domain. The disturbance input is assumed to be a zero mean white noise stationary random process. The $Z$ transform of the system response, $Y^{i}(z)$, can be written as follows

$$
Y^{i}(z)=T_{i \epsilon}(z) X(z),
$$

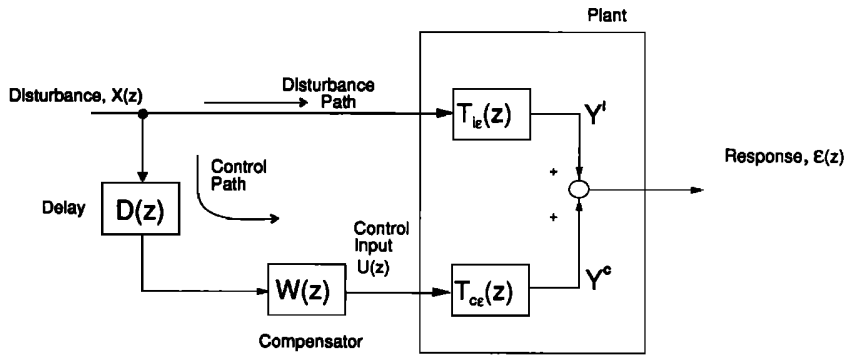

FIG. 2. Discrete time domain feedforward block diagram with a delay in the control path.

where $T_{i \epsilon}(z)$ is the transfer function between the disturbance input and the error output, and $X(z)$ is the $Z$ transform of the input signal.

The system is controlled by feeding forward a reference signal, coherent to the disturbance, through the control filter $W(z)$. The reference signal is obtained by tapping the disturbance input $X(z)$ and passing it through a delay filter $D(z)$ which is given by

$$
D(z)=z^{-d} \text {. }
$$

The time delay produced by the filter $D(z)$ is $\tau=d t_{s}$, where $t_{s}$ is the sampling period that is related to the sampling frequency $f_{s}$ by $t_{s}=1 / f_{s}$. The time delay $\tau$ represents the difference in propagation time of a pulse through the control and disturbance paths to the error sensor. The control system is causal by selecting the delay $d=0$, i.e., the disturbance takes the same time, in this ideal situation, to propagate through the control and disturbance paths. The control system is made noncausal by setting the delay parameter positive, $d>0$.

The analysis in the discrete time-domain allows us to define a physically realizable causal FIR filter as the compensator $W(z)$. That is

$$
W(z)=\sum_{l=0}^{L} w_{z^{-l}}
$$

where $w_{l}$ is the $l$ th weight; and $(L+1)$ is the filter size equal to the number of weights.

The $Z$ transform of the response, $Y^{c}(z)$, due to the control input is given as follows

$$
Y^{c}(z)=T_{c \epsilon}(z) U(z),
$$

where $T_{c \epsilon}(z)$ is the transfer function between the control input and the error output, and $U(z)$ is the $Z$ transform of the control input.

The response of the system can then be computed as the superposition of the response due to the disturbance and control inputs as follows

$$
\epsilon(z)=\left[T_{i \epsilon}(z)+D(z) W(z) T_{c \epsilon}(z)\right] X(z) .
$$

In feedforward control, the optimum filter weights that define the control input signal are determined by minimizing the mean square value of the error output. The mean square value of $\epsilon(z)$ can be computed as ${ }^{19}$ 


$$
\sigma^{2}=\frac{1}{2 \pi j} \oint \Phi_{\epsilon \epsilon}(z) \frac{d z}{z},
$$

where $\Phi_{\epsilon \epsilon}(z)$ is the discrete power spectral density function (PSDF) of the error sequence; $j$ is the imaginary number; and the path of integration is counterclockwise on a circle centered at the origin on the $z$ plane.

\section{A. Frequency-domain analysis}

The integration in Eq. (6) can be conveniently solved in the frequency domain by using the transformation ${ }^{19}$

$$
z=e^{j \omega t_{s}} \text {, }
$$

which relates the discrete time variable $z$ to the nonnormalized frequency $\omega$. Using Eq. (7), the integral in Eq. (6) becomes ${ }^{19}$

$$
\sigma^{2}=\frac{1}{2 \pi} \int_{-\omega_{N y}}^{\omega_{N y}} \Phi_{\epsilon \epsilon}\left(e^{j \omega t_{s}}\right) d \omega
$$

where $\Phi_{\epsilon \epsilon}(\omega)$ is the error signal PSDF defined in the frequency domain, and $\omega_{N y}=\pi / t_{s}$ is the Nyquist circular frequency. The same transform, Eq. (7), applied to Eq. (5) gives the frequency content of the error output as

$\epsilon(\omega)=\left[T_{i \epsilon}\left(e^{j \omega t_{s}}\right)+D\left(e^{j \omega t_{s}}\right) W\left(e^{j \omega t_{s}}\right) T_{c \epsilon}\left(e^{j \omega t_{s}}\right)\right] x(\omega)$.

Replacing Eq. (7) into Eqs. (2) and (3) and these into Eq. (9) results in:

$\epsilon(\omega)=\left[T_{i \epsilon}\left(e^{j \omega t_{s}}\right)+e^{-j \omega d t_{s}}\{W\}^{T}\{f\} T_{c \epsilon}\left(e^{j \omega t_{s}}\right)\right] x(\omega)$,

where

$$
\{\boldsymbol{W}\}=\left\{w_{0}, w_{1}, w_{2}, \ldots, w_{L}\right\}^{T}
$$

is the vector of filter weights, and

$$
\{f\}=\left\{1, e^{-j \omega t_{s}}, \ldots, e^{-j \omega L t_{s}}\right\}^{T}
$$

is known as the complex sinusoid vector in digital spectral analysis; ${ }^{20}$ and the vector transpose is denoted by the superscript $T$.

The power spectral density function of the error signal is now defined by

$$
\Phi_{\epsilon \epsilon}(\omega)=E\left[\epsilon(\omega) \epsilon(\omega)^{*}\right],
$$

where $E[\cdot]$ is the expected value operator; and the asterisk denotes the complex conjugate. Substituting Eq. (10) into Eq. (13) yields

$$
\begin{aligned}
\Phi_{\epsilon \epsilon}(\omega)= & \mid T_{i \epsilon}\left(e^{j \omega t_{s}}\right) \\
& \left.+e^{-j \omega d t_{s}\{W}\right\}\left.^{T}\{f\} T_{c \epsilon}\left(e^{j \omega t_{s}}\right)\right|^{2} \Phi_{x x}(\omega) .
\end{aligned}
$$

Since the disturbance is a zero mean white noise random process, the disturbance PSDF is constant, $\Phi_{x x}(\omega)=\Phi_{0}$. The mean square value of the error signal as a function of the filter weights can now be computed by replacing Eq. (14) into Eq. (8), and expanding the complex modulus to yield

$$
\sigma^{2}=\sigma_{i}^{2}+\{W\}^{T}[R]\{W\}+\{W\}^{T}\left(\{H\}+\{H\}^{*}\right),
$$

where

$$
\sigma_{i}^{2}=\frac{1}{2 \pi} \int_{-\omega_{N y}}^{\omega_{N y}}\left|T_{i \epsilon}\left(e^{j \omega t_{s}}\right)\right|^{2} \Phi_{0} d \omega
$$

is the variance of the system response due to the disturbance input alone; matrix $[R]$ given by

$$
[R]=\left[\begin{array}{ccccc}
R(0) & R(1) & R(2) & \ldots & R(L) \\
R(-1) & R(0) & R(1) & & \vdots \\
R(-2) & R(-1) & R(0) & & \\
\vdots & & & \ddots & R(1) \\
R(-L) & \ldots & & R(-1) & R(0)
\end{array}\right]
$$

is a Hermitian Toeplitz matrix that is formed from the $(L+1)$ coefficients

$$
R(r)=\frac{1}{2 \pi} \int_{-\omega_{N y}}^{\omega_{N y}}\left|T_{c \epsilon}\left(e^{j \omega t_{s}}\right)\right|^{2} e^{j \omega r_{s}} \Phi_{0} d \omega
$$

and the vector $\{H\}$ is given by

$$
\{H\}=\{H(0), H(1), \ldots, H(L)\}^{T}
$$

with

$$
\begin{aligned}
H(r)= & \frac{1}{2 \pi} \int_{-\omega_{N y}}^{\omega_{N y}} T_{i \epsilon}\left(e^{-j \omega t_{s}}\right) T_{c \epsilon}\left(e^{j \omega t_{s}}\right) \\
& \times e^{-j \omega(d+r) t_{s}} \Phi_{0} d \omega .
\end{aligned}
$$

In adaptive feedforward control the error output mean square value in Eq. (15), referred as the performance surface, is a quadratic function of the filter weights $w_{l}$. The optimum weights are those that minimize the mean square value of the error signal. Thus, differentiating Eq. (15) with respect to $w_{l}$ and setting the result to zero yields the following linear system of equations

$$
[R]\{W\}=-\left\{H_{r}\right\},
$$

where $\left\{H_{r}\right\}$ is the real part of the complex vector $\{H\}$.

The properties of matrix $[R]$ will be described in further detail in the next section. Assuming now that the matrix $[R]$ is nonsingular, solving for the optimum vector $\{W\}$ in Eq. (21), and replacing it back into Eq. (15) yields the minimum mean square value as

$$
\left(\sigma^{2}\right)_{\min }=\sigma_{i}^{2}-\left\{H_{r}\right\}^{T}[R]^{-1}\left\{H_{r}\right\} .
$$

The minimum mean square value of the controlled output response is the difference between the mean square value due to the disturbance alone and the quadratic form $\left\{H_{r}\right\}^{T}[R]^{-1}\left\{H_{r}\right\}$. Thus, the control system performance will be completely determined by the properties of this quadratic form.

The effectiveness of the control system can be measured by the performance ratio

$$
\eta(\mathrm{dB})=10 \log _{10}\left(1-\frac{\left\{H_{r}\right\}^{T}[R]^{-1}\left\{H_{r}\right\}}{\sigma_{i}^{2}}\right)
$$


(a)

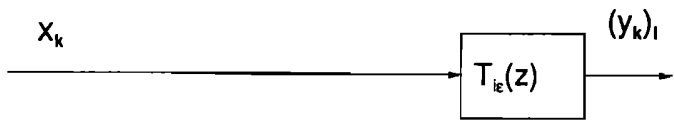

(b)

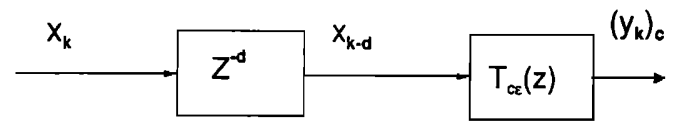

FIG. 3. Random processes associated to the integrals in Eqs. (16), (18), and (20).

that gives the error output power reduction in decibels.

\section{CONTROL SYSTEM PERFORMANCE ANALYSIS}

A physical interpretation for the terms in matrix $[R]$ and vector $\{H\}$ can be derived by considering the two zero mean random processes depicted in Fig. 3. The process in Fig. 3(a) is the response of the system $\left(y_{k}\right)_{i}$ when excited at the disturbance location by the sequence $x_{k}$, while the process in Fig. 3(b) is the response of the system $\left(y_{k}\right)_{c}$ when excited at the control location by the delayed sequence $x_{k-d}$. The term $R(r)$ represents the autocorrelation sequence of the response $\left(y_{k}\right)_{c}$ as ${ }^{20}$

$R(r)=E\left[\left(y_{k}\right)_{c}\left(y_{k-r}\right)_{c}\right]=\frac{1}{2 \pi} \int_{-\omega_{N y}}^{\omega_{N y}} \Phi_{y_{c} y_{c}}(\omega) e^{j \omega r t_{s}} d \omega$,

where the PSDF in Eq. (24) is given in terms of the disturbance PSDF and the system dynamics as

$$
\Phi_{y_{c} y_{c}}(\omega)=\left|T_{c \epsilon}\left(e^{j \omega t_{s}}\right)\right|^{2} \Phi_{0} .
$$

Since the autocorrelation sequence $R(r)$ is independent of the delay parameter $d$, the properties of the autocorrelation matrix of the random process $\left(y_{k}\right)_{c}$ will also be independent of the delay. The properties of $[R]$ are a function of the system dynamics, through the control input and error output transfer function $T_{c \epsilon}(z)$, and the sampling frequency. Since the autocorrelation function of a real stationary random process is an even function $R(r)=R(-r)$, the matrix $[R]$ is real and symmetric. Because of the nonnegative property of the autocorrelation function, the autocorrelation matrix is positive semidefinite. ${ }^{20}$

The term $H(r)$ in Eq. (20) represents the crosscorrelation sequence between the random processes $\left(y_{k}\right)_{i}$ and $\left(y_{k}\right)_{c}$ as follows ${ }^{20}$

$$
\begin{aligned}
H(r) & =E\left[\left(y_{k}\right)_{i}\left(y_{k-r-d}\right)_{c}\right] \\
& =\frac{1}{2 \pi} \int_{-\omega_{N y}}^{\omega_{N y}} \Phi_{y_{j} y_{c}}(\omega) e^{-j \omega r_{s}} d \omega,
\end{aligned}
$$

where the cross PSDF in Eq. (26) is given by

$$
\Phi_{y_{y} y_{c}}(\omega)=T_{i \epsilon}\left(e^{-j \omega t_{s}}\right) T_{c \epsilon}\left(e^{j \omega t_{s}}\right) e^{-j \omega d t_{s}} \Phi_{0} .
$$

Unlike the autocorrelation, the cross-correlation sequence of a real random process is a complex variable. From Eq. (27), $H(r)$ is a function of the system dynamics

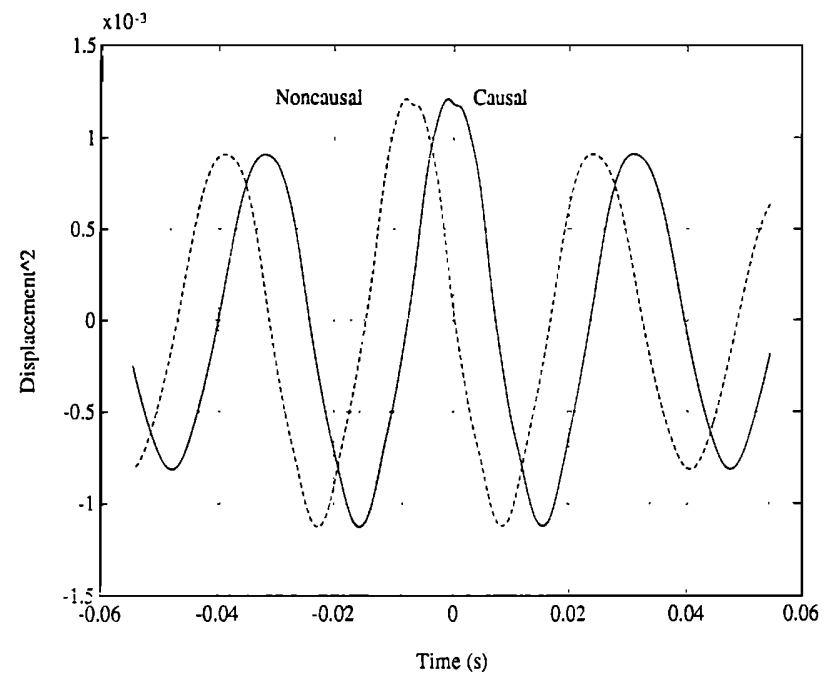

FIG. 4. Real part of cross-correlation sequence $H(r)$ in Eq. (26) for a typical structure.

through the transfer functions $T_{i \epsilon}(z)$ and $T_{c \epsilon}(z)$, the selected sampling frequency and the control path delay $d$. To graphically illustrate the dependance of $H(r)$ with the delay parameter $d$, the real part of the cross-correlation sequence is plotted in Fig. 4 as a continuous line for a causal system by setting $d=0$. Assuming the control path has a delay $d$, the cross-correlation sequence for the noncausal system is the same cross-correlation of the causal system shifted toward the left as shown in Fig. 4 with dashed lines.

Important qualitative conclusions on the controlled system performance can be drawn by considering the special case of sinusoidal random process. ${ }^{20}$ In this process, a random sequence is approximated as the sum of $M$ real sinusoids as

$$
x_{k}=\sum_{m=1}^{M} A_{m} \sin \left(\omega_{m} k t_{s}+\theta_{m}\right),
$$

where $A_{m}$ is the real amplitude of the $m$ th sinusoid of circular frequency $\omega_{m}$, and the associated phase $\Theta_{m}$ is a uniformly distributed random variable on the interval 0 to $2 \pi$. The amplitude $A_{m}$ is related to the magnitude of the PSDF at frequency $\omega_{m}$. The number and frequency of the sinusoids can be arbitrary. For the sake of clarity in the presentation, we select a uniform distribution for the sinusoid frequencies as $\omega_{m}=m(\Delta \omega)$ that results in $A_{m}=2\left(\Phi_{0} \Delta \omega\right)^{1 / 2}$. In the limiting case that $M \rightarrow \infty$ and $\Delta \omega \rightarrow 0$ the sequence in Eq. (28) will be a true white noise random sequence.

Replacing Eq. (28) into the process described in Fig. 3 (b), it is not difficult to show that the autocorrelation sequence of this sinusoidal process can be easily computed in closed form $\mathrm{as}^{20}$

$$
R(r)=\Phi_{0} \Delta \omega \sum_{m=1}^{M}\left|T_{c \epsilon}\left(\omega_{m}\right)\right|^{2} \cos \left(\omega_{m} r t_{s}\right)
$$

and the associated autocorrelation matrix becomes 


$$
[R]=\Phi_{0} \Delta \omega \sum_{m=1}^{M}\left|T_{c \epsilon}\left(\omega_{m}\right)\right|^{2}\left\{S_{m}\right\}\left\{S_{m}\right\}^{T},
$$

where $\left\{S_{m}\right\}$ is

$$
\left\{S_{m}\right\}=\left[1, \cos \left(\omega_{m} t_{s}\right), \cos \left(\omega_{m} 2 t_{s}\right), \ldots, \cos \left(\omega_{m} L t_{s}\right)\right]^{T} .
$$

Again the autocorrelation matrix $[R]$ is real, symmetric and in general positive semidefinite, or in other words the eigenvalues of matrix $[R]$ are real and non-negative. The rank of the autocorrelation matrix can be shown to be $2 M .^{20}$ The dimension of matrix $[R]$ is equal to the number of filter weights, $(L+1)$. If $2 M$ is greater or equal to ( $L$ +1 ), the eigenvalues of $[R]$ are all real and positive and the matrix inverse in Eq. (22) exists. On the other hand, if $(L+1)$ is greater than $2 M$, there will be $(L+1)-2 M$ zero eigenvalues and matrix $[R]$ is singular. A physical interpretation can be gained by considering the case where the number of weights in the filter is twice the number of sinusoids included in the process, $(L+1)=2 M$. Thus, there are enough weights to adjust the phase and magnitude of each sinusoid to drive the output mean square value to zero, $\left(\sigma^{2}\right)_{\min }=0$. Clearly, increasing the number of filter weights provides no further reduction and this is mathematically depicted by the rank of $[R]$ being $2 M$.

For the case of $(L+1)<2 M$, the positive definite property of $[R]$ and of its inverse allows us to write

$$
\left\{H_{r}\right\}^{T}[R]^{-1}\left\{H_{r}\right\}>0
$$

for any arbitrary vector $\left\{H_{r}\right\}$, which has the important implication that there will always be some degree of reduction in the output variance in Eq. (22) in spite of any delay in the control path. Because of the positive definite property, it is not difficult to show that the quadratic form in Eq. (32) is a monotonically increasing function. That is

$$
\left(\left\{H_{r}\right\}^{T}[R]^{-1}\left\{H_{r}\right\}\right)_{L+1} \geqslant\left(\left\{H_{r}\right\}^{T}[R]^{-1}\left\{H_{r}\right\}\right)_{L} .
$$

Therefore, the deterioration in the control performance due to the time delay could at least be partially compensated by increasing the filter size. This phenomenon was observed in the numerical and experimental studies of the next section.

\section{EXPERIMENTAL AND NUMERICAL RESULTS}

Both analytical and experimental investigations of the system causality for broadband structural control were first performed on a simply supported beam. The beam is made of steel and has a length of $L=380 \mathrm{~mm}$. Two thin, flexible metal shims connect the beam to a rigid frame, providing

TABLE I. Simply supported beam natural frequencies.

\begin{tabular}{ccc}
\hline \hline & \multicolumn{2}{c}{ Natural frequency $(\mathrm{Hz})$} \\
\cline { 2 - 3 } & Analytical & Experimental \\
\hline 1 & 31.7 & 32.9 \\
2 & 127.0 & 126.9 \\
3 & 285.6 & 282.3 \\
4 & 507.2 & 505.5 \\
\hline \hline
\end{tabular}

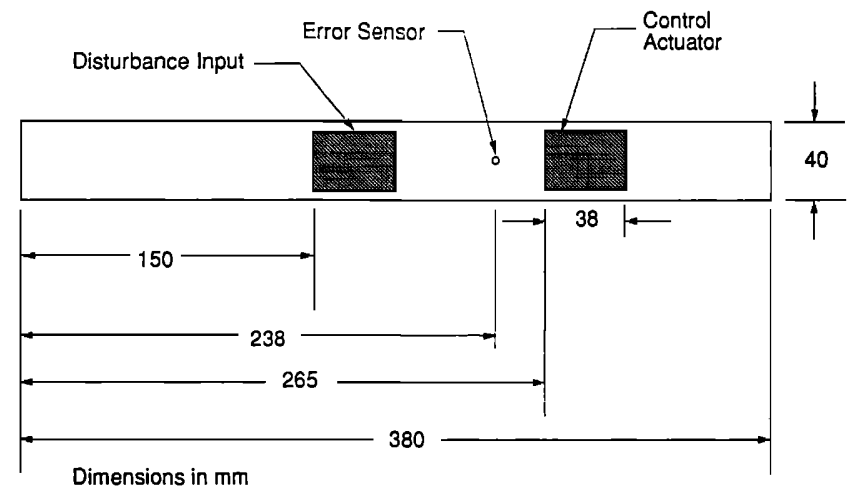

FIG. 5. Sensor and actuator locations on the simply supported experimental beam analysis.

the simply supported end conditions. The experimentally measured natural frequencies are presented in Table I. Figure 5 shows the location of the error sensor and disturbance and control inputs. The error sensor was a miniaccelèrometer located at $x_{e}=0.626 L$. The control actuation was applied by a co-located set of piezoelectric patches mounted on each side of the beam surfaces wired out-of-phase to produce a pair of bending moments applied at $x_{c 1}=0.797 \mathrm{~L}$ and $x_{c 2}=0.697 \mathrm{~L}$. The disturbance input action was also applied by a piezoelectric patch. The resulting disturbance bending moments are applied at $x_{d 1}$ $=0.494 L$ and $x_{d 2}=0.394 L$. The experiment was limited to control the first three bending modes because the location of the control actuator pair rendered the fourth mode uncontrollable. Consequently, the cutoff frequency of the low-pass filters used in the experimental setup was set at $400 \mathrm{~Hz}$.

A schematic diagram of the complete experimental setup is shown in Fig. 6. A white noise signal was generated by a Brüel \& Kjær 2032 spectrum analyzer, which was low-pass filtered to obtain band-limited random noise from 0 to $400 \mathrm{~Hz}$. This signal was input into a power amplifier whose output drove the disturbance piezoelectric patch. The same input signal was fed into the controller. The control system used in the experiment is a broadband adaptive LMS controller implemented on a Texas Instru-

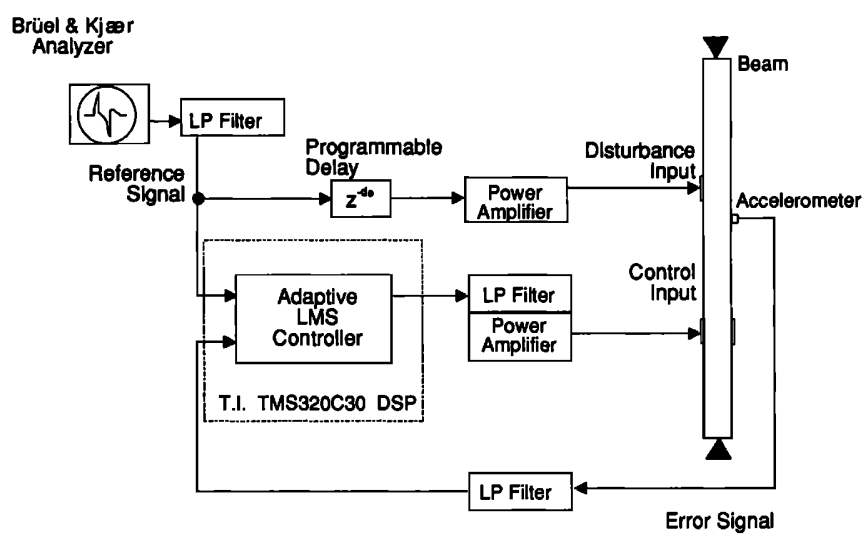

FIG. 6. Experimental setup for testing the feedforward broadband control algorithm. 
ment TMS320C30 digital signal processor (DSP). ${ }^{16}$ The sampling rate used for the control experiments was $f_{s}$ $=2000 \mathrm{~Hz}$. The control signal from the DSP was low-pass filtered and fed into a power amplifier that in turn drove the control piezoelectric patches.

The propagation time from both the disturbance and control inputs to the error sensor was measured by computing the cross-correlation functions across each path while being excited by white noise. The propagation time for each path is found at the largest peak on the respective cross-correlation function. It was found that the control path has an average delay time of $2.7 \mathrm{~ms}$ with respect to the disturbance path, which was introduced mainly by the smoothing filter in the control path. Therefore, the experimental setup yields a noncausal control system. A programmable delay $z^{-d_{e}}$ was installed in the disturbance path allowing the control system to be made causal by varying the delay $d_{e}$. The reader should not confuse this experimental delay parameter $d_{e}$ with the analytical delay parameter $d$ used in Secs. I and II. The delay in the disturbance path in the experimental setup simply allow us to compare the behavior of the causal with the noncausal control system. On the other hand, the analytical delay parameter $d$ in the control path has the purpose of modeling the actual time delay in a control system whose characteristics are being investigated. It is important to mention that in a real implementation, delaying the disturbance signal as in this experiment is seldom possible. Thus, it is of a paramount importance to address the performance of real noncausal systems.

An analytical model of the experimental setup was also derived by solving the eigenvalue problem of a simply supported beam. The bending stiffness is $E I=5.329 \mathrm{NT} \mathrm{m}^{2}$ and mass per unit length is $m=0.626 \mathrm{NT} \mathrm{s}^{2} / \mathrm{m}^{2}$ that corresponds to the experimental beam properties. The system is assumed to have $0.2 \%$ viscous damping in each mode. Again only the three first modes are included in the response analysis. The well known natural frequencies and mass normalized mode shapes are given by

$$
f_{n}=\frac{1}{2 \pi}\left(\frac{n \pi}{L}\right)^{2} \sqrt{\frac{E I}{m}} ; \quad \Phi_{n}(x)=\sqrt{\frac{2}{m L}} \sin \left(\frac{n \pi x}{L}\right) .
$$

The analytical natural frequencies are also tabulated in Table I and they agree very well with the experimentally measured ones. These eigenproperties are used to compute the transfer functions $T_{i \epsilon}(z)$ and $T_{c \epsilon}(z)$ needed for the analysis. The derivation of these transfer function is detailed in the Appendix.

\section{A. Causal control system}

The behavior of the causal control system was first investigated by implementing a delay of $3 \mathrm{~ms}\left(d_{e}=6\right)$ into the disturbance path in the experimental setup. This experimental delay parameter is nearly equivalent to a delay parameter $d=0$ in the analysis. Figure 7 shows a comparison of the measured error signal before and after control using an adaptive FIR filter with 24 weights. Although the error was not completely canceled, a large amount of vibration attenuation was achieved by the controller. A com-
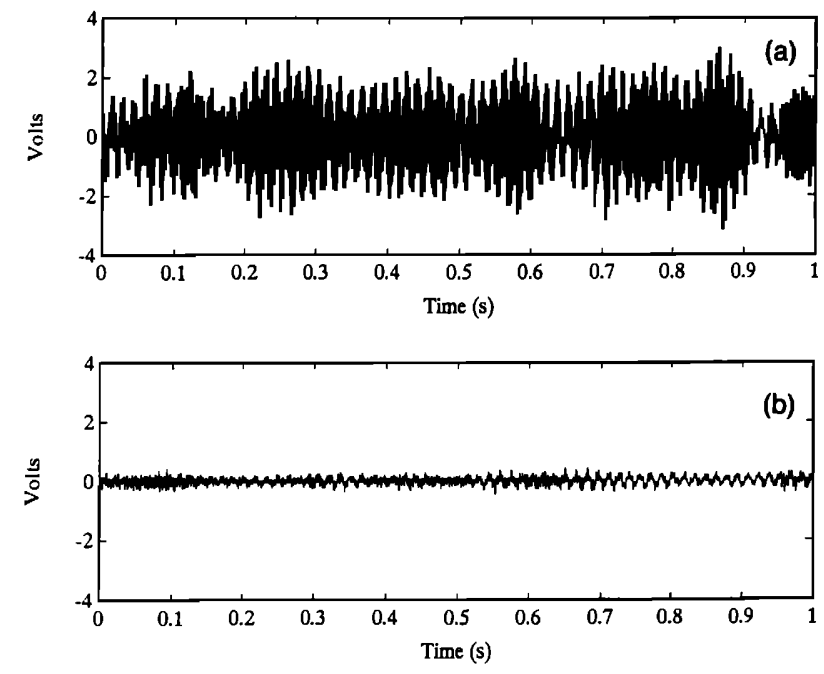

FIG. 7. Error signal from the structure using a FIR filter with 24 weights. (a) Before control; (b) after control.

parison of the spectrum of the error signal before and after control is shown in Fig. 8, and it gives additional insight into the control mechanism. Despite an increase of the spectrum in some off-resonance frequencies bands, the spectrum at the resonances $(32.9,126.9$, and $282.3 \mathrm{~Hz})$ displays a reduction of approximately $20-25 \mathrm{~dB}$. The result was a net reduction of the mean-square error of $19.4 \mathrm{~dB}$. Thus, the controller behaves as a wideband controller rather than broadband controller, attenuating the large frequency components that occur near the structural resonances while adding energy at the antiresonances.

The influence of the adaptive filter size on the controller performance was then investigated experimentally by varying the filter size $(L+1)$. The reductions in the error signal power was measured for $12,14,16,24,32,48,64$, and 80 weights in the FIR filter and they are plotted in Fig. 9. It can be seen that the control performance quickly improves with larger filters to reach a nearly constant reduction of $20 \mathrm{~dB}$. The analytical response of the beam for a causal system is obtained by setting the delay parameter

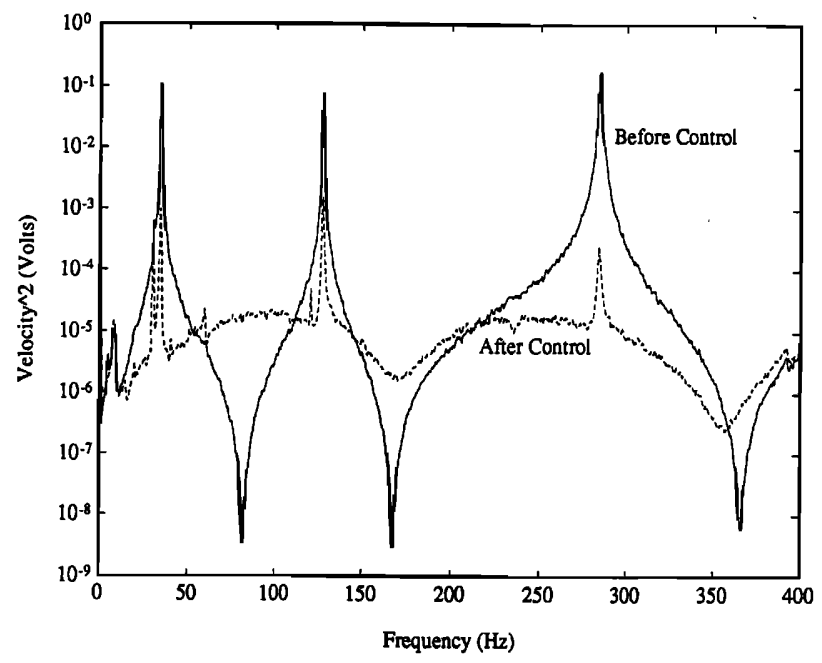

FIG. 8. Spectrum of error signal using a FIR filter with 24 weights. 


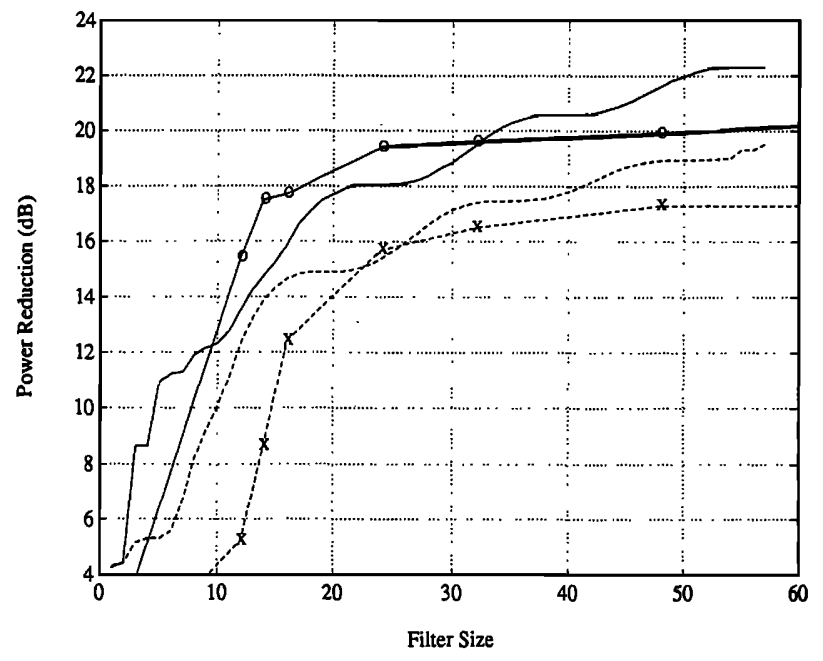

FIG. 9. Power reduction of error signal as a function of the filter size. Analytical causal; ----- analytical noncausal; $\mathrm{O}-\mathrm{O}$ experimental causal; $\times---X$ experimental noncausal system.

$d=0$ in Eq. (2). The integrals in Eqs. (16), (18), and (20) are numerically solved as described in the Appendix. The power reduction at the error sensor location computed from Eq. (23) is plotted in Fig. 9 as a function of the filter size. The analytical prediction shows the same general trend as the experimental observations with two exceptions. Firstly, the analytical curves display some oscillation in the monotonically increasing performance function which is not observed in the experiment. The reason for this discrepancy may lay in the lack of resolution in the experimental results. The period of this oscillation is related to the period of the first natural mode of the beam. The same oscillatory phenomenon was also observed by Nelson et al. ${ }^{17}$ Secondly, the analytical model displays better control for larger filter sizes than in the experimental observations. This is probably due to the fact that the controller may not have completely converged at the time the response was measured since large filters requires longer convergence times. In addition the convergence parameter was set very small $\left(10^{-7}\right)$ for the large filter size, thus increasing the effects of round off error.

\section{B. Noncausal control system}

The behavior of the noncausal control system was then studied by setting the programmable delay $d_{e}$ to zero in the experimental setup of Fig. 6. This is equivalent to setting $d=6$ in the analysis. The experimental error signal time histories and spectrums before and after control show the same features as for the causal system in Figs. 7 and 8, and thus they are not presented here. The reduction in the error signal power in terms of the FIR filter size is again plotted in Fig. 9. The analytically predicted power reduction for the noncausal control system is also plotted in Fig. 9. Both the analytical and experimental results show that the effectiveness of the noncausal controller is severely compromised for filters with less than 24 weights compared with the causal controller. However, for larger filter size, the

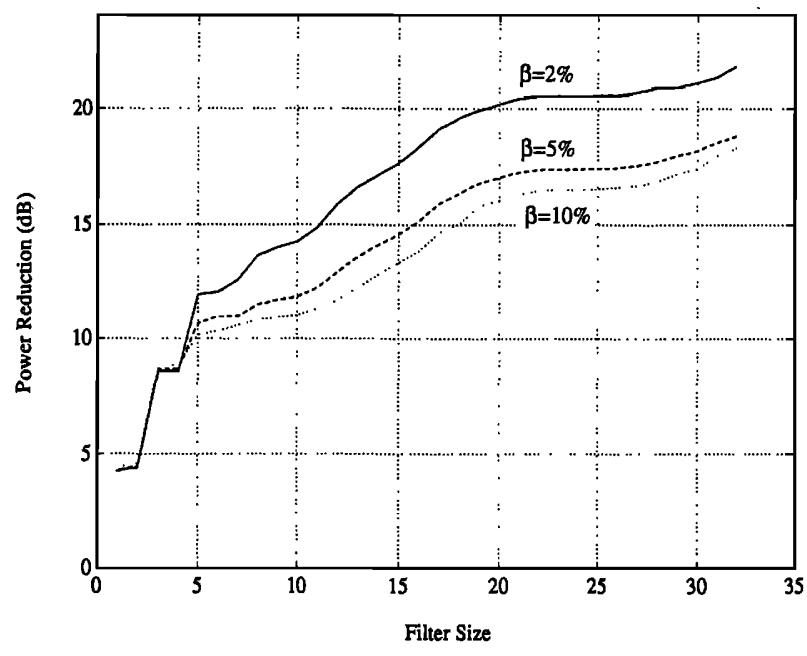

FIG. 10. Power reduction of error signal as function of the filter size and modal damping for a causal system.

noncausal controller shows significant reduction of the error variance which is only about $3 \mathrm{~dB}$ below the causal controller.

The good agreement between the analytical and experimental results, for both the causal and noncausal control systems, validates the proposed formulation as an analytical tool to predict control system performance. This simple laboratory demonstration shows that the formulation presented here can be used to predict the controller performance as a function of the system parameters such as delay time, sampling frequency, filter size, number of modes to control, etc. As an example, it is useful to predict the minimum filter size to achieve a required degree of reduction of the error signal. The causal controller of the experimental setup needed 12 coefficients to reduce the error variance by $15 \mathrm{~dB}$. On the other hand, the noncausal controller, with a delay of $3 \mathrm{~ms}$, requires 24 filter weights for the same reduction. However, increasing the filter size also slows the adaptive process and increases the computational effort. Thus, careful analysis of the control performance should be considered before a controller is implemented on a particular application.

\section{Effect of damping}

The effect of damping in a causal system is investigated by varying the modal viscous damping ratio $\beta$ from $2 \%$ to $10 \%$. As shown in Fig. 10 the decrease in damping results in better attenuation of the error output mean square value. This is due to the enhanced notch filtering effect of the structure that yields the response to resemble the superposition of multiple sinusoids whose frequencies are the natural frequencies of the structure. This behavior is described by Nelson et al. ${ }^{17}$ as an increase in the "predictability" of the broadband input by the filtering effect of the structure. In the limiting case of the modal damping approaching zero, a filter with the number of coefficients equal to twice the number of modes to control would be sufficient to completely cancel the error signal. Figure 11 presents the parametric study of damping in the noncausal 


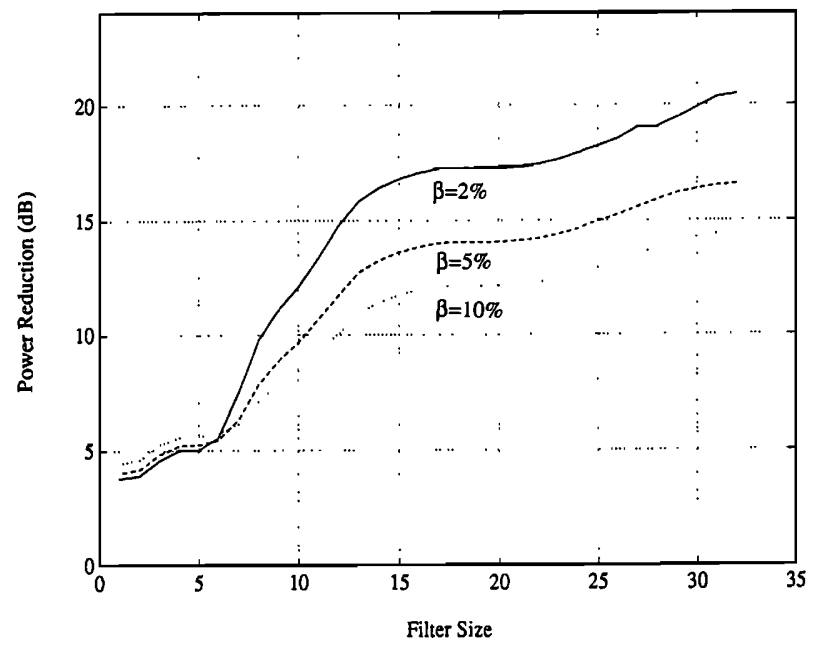

FIG. 11. Power reduction of error signal as function of the filter size and modal damping for a noncausal system.

system. The results shows the same general deterioration in the performance with the increase in damping as for the causal system. The analytical formulation developed here can also be used to investigate the variation in the controller performance with different delays and spectral content of the broadband input disturbance.

\section{CONCLUSIONS}

A formulation was developed to carried out causality analysis of feedforward controlled elastic systems subjected to broadband excitations. The control approach used is the feedforward control approach with a FIR filter as compensator. The proposed analytical tool was developed in the frequency-domain and can be used to predict the performance of this type of controllers in terms of system parameters such as delay time, damping, spectral content of the input, filter size, etc... It is demonstrated that reduction in the error signal mean square value is always achievable for any noncausal control system. The analysis also shows that the deterioration in the control performance due to the delay in the control path can be at least partially compensated by increasing the compensator order. The applicability of the formulation is demonstrated on a laboratory model of a simply supported finite beam. The control performance was investigated for both causal and noncausal systems as a function of the compensator size and system damping. The numerical results are also corroborated experimentally. Good agreement is observed between the numerically predicted and measured results, thus validating the proposed formulation.

\section{ACKNOWLEDGMENT}

The authors gratefully acknowledge the support of this work by the Office of Naval Research Grant No. ONRN00014-92-J-1170.

\section{APPENDIX}

The integrals in Eqs. (16), (18), and (20) do not have closed form solution. A direct numerical integration poses some difficulties because the peaked nature of the typical structural transfer functions and also due to the oscillatory behavior of the term $e^{j \omega r t}$ in Eqs. (18) and (20). Here a detailed description is presented for the numerical evaluation of these integrals.

The transfer function between the control input and error sensor can be written as follows

$$
T_{c \epsilon}(z)=\sum_{n=1}^{N} u_{n} \epsilon_{n} H_{n}(z),
$$

the modal control force $u_{n}$ and the modal error components $\epsilon_{n}$ are given by

$$
u_{n}=\frac{d \phi_{n}\left(x_{c 2}\right)}{d x}-\frac{d \phi_{n}\left(x_{c 1}\right)}{d x} ; \quad \epsilon_{n}=\phi_{n}\left(x_{e}\right) \text {. }
$$

$N$ is the number of modes included in the analysis; and

$$
H_{n}(z)=\frac{b_{n 1} z}{z-a_{n 1} z+a_{n 2}}
$$

is the $n$th modal frequency response function (FRF) defined in the discrete domain. The coefficients $b_{n 1}, a_{n 1}$, and $a_{n 2}$ are related to the $n$th natural frequency $\omega_{n}$, damping ratio $\beta_{n}$, and the sampling period $t_{s}$ as $^{21}$

$$
\begin{aligned}
& b_{n 1}=\frac{e^{-\beta_{n} \omega_{n} t_{s}}}{\omega_{n}\left(1-\beta_{n}^{2}\right)} \sin \left(\omega_{n d}\right) ; \\
& a_{n 1}=2 e^{-\beta_{n} \omega_{n} t_{s}} \cos \left(\omega_{n d}\right) ; \\
& a_{n 2}=e^{-2 \beta_{n} \omega_{n} t_{s}}
\end{aligned}
$$

with $\omega_{n d}=\omega_{n}\left(1-\beta^{2}\right)^{1 / 2}$. Solving for the roots of the denominator in Eq. (A3), $H_{n}(z)$ can be written as

$$
H_{n}(z)=\frac{b_{n 1} z}{\left(z-z_{n}\right)\left(z-z_{n}^{*}\right)},
$$

where the roots are

$$
z_{n}=e^{-\beta_{n} \omega_{n} t_{s}}\left\{\cos \left(\omega_{n d} t_{s}\right)+j \sqrt{1-\cos ^{2}\left(\omega_{n d} t_{s}\right)}\right\} .
$$

The modules square of the transfer function $T_{c \epsilon}(z)$ is computed as

$$
\begin{aligned}
\left|T_{c \epsilon}(z)\right|^{2} & =T_{c \epsilon}(z) T_{c \epsilon}\left(z^{-1}\right) \\
& =\sum_{n=1}^{N} \sum_{m=1}^{N} u_{n} u_{m} \epsilon_{n} \epsilon_{m} H_{n}(z) H_{m}\left(z^{-1}\right)
\end{aligned}
$$

where by replacing $z$ by $z^{-1}$ is equivalent to take the complex conjugate in the frequency domain. Replacing Eq. (A5) into (A7) and using partial fraction expansions, the product of the modal FRFs can be expanded as follows

$$
H_{n}(z) H_{m}\left(z^{-1}\right)=\frac{A_{n m}}{z-z_{n}}+\frac{B_{n m}}{z-z_{n}^{*}}+\frac{C_{n m}}{z-1 / z_{m}}+\frac{D_{n m}}{z-1 / z_{m}^{*}},
$$

where the coefficients in the partial fractions are easily computed. Replacing Eq. (A8) into (A7) becomes 


$$
R(r)=\frac{1}{2 \pi} \sum_{n=1}^{N} \sum_{m=1}^{N} u_{n} u_{m} \epsilon_{n} \epsilon_{m} I_{n m}(r),
$$

where

$$
\begin{aligned}
I_{n m}(r)= & A_{n m} \int_{-\omega_{N y}}^{\omega_{N y}} \frac{e^{j \omega r t_{s}}}{e^{j \omega t_{s}}-z_{n}} d \omega \\
& +B_{n m} \int_{-\omega_{N y}}^{\omega_{N y}} \frac{e^{j \omega r t_{s}}}{e^{j \omega t_{s}}-z_{n}} d \omega \\
& +C_{n m} \int_{-\omega_{N y}}^{\omega_{N y}} \frac{e^{j \omega r t_{s}}}{e^{j \omega t_{s}}-1 / z_{m}} d \omega \\
& +D_{n m} \int_{-\omega_{N y}}^{\omega_{N y}} \frac{e^{j \omega r t_{s}}}{e^{j \omega t_{s}}-1 / z_{m}^{*}} d \omega .
\end{aligned}
$$

Now each integral in Eq. (A9) is better conditioned that the original integral in Eq. (18). The numerical solution of the integrals in Eq. (A9) does not pose any problems for wide range of the parameters $r$ and $t_{s}$. Similarly, the integral in Eq. (16) becomes

$$
\sigma_{d}^{2}=\frac{1}{2 \pi} \sum_{n=1}^{N} \sum_{m=1}^{N} f_{n} f_{m} \epsilon_{n} \epsilon_{m} I_{n m}(0)
$$

and Eq. (20)

$$
H(r)=\frac{1}{2 \pi} \sum_{n=1}^{N} \sum_{m=1}^{N} f_{n} u_{m} \epsilon_{n} \epsilon_{m} I_{n m}(d+r),
$$

where the disturbance modal force is given by

$$
f_{n}=\frac{d \phi_{n}\left(x_{d 2}\right)}{d x}-\frac{d \phi_{n}\left(x_{d 1}\right)}{d x}
$$

${ }^{1}$ B. Widrow, J. R. Glover, Jr., J. M. McCool, J. Kaunitz, C. S. Williams, R. H. Hearn, J. R. Zeidler, E. Dong, Jr., and R. C. Goodlin, "Adaptive Noise Canceling: Principles and Applications," Proc. IEEE 63, 16921716 (1975).

${ }^{2}$ G. E. Warnaka, "Active Attenuation of Noise: the State of the Art," Noise Control Eng. 18, 100-110 (1982).

${ }^{3}$ R. J. Silcox and H. C. Lester, "An Evaluation of Active Control in a Cylindrical Shell," J. Vib. Stress, Reliability Design 111, 337-342 (1989).
${ }^{4}$ C. R. Fuller, “Active Control of Sound Radiation from a Vibrating Rectangular Panel by Sound Sources and Vibration Inputs: an Experimental Comparison," J. Sound Vib. 145, 195-215 (1991).

${ }^{5}$ W. Redman-While, P. A. Nelson, and A. R. D. Curtis, "Experiments on Active Control of Flexural Wave Power," J. Sound Vib. 112, 181187 (1987).

${ }^{6}$ A. H. Von Flowtow and B. Schafer, "Wave-absorbing Controller for a Flexible Beam," J. Guidance, Control Dynam. 9, 673-680 (1986).

${ }^{7} \mathrm{~J}$. Scheuren, "Active Attenuation of Bending Waves in Beams," Proc. Inst. Acoust. 12, 623-629 (1990).

${ }^{8}$ G. P. Gibbs and C. R. Fuller, "Experiments on Active Control of Vibrational Power Flow Using Piezoceramics Actuators and Sensors," AIAA paper 90-1132 (1990).

${ }^{9}$ C. R. Fuller, G. P. Gibbs, and R. J. Silcox, "Simultaneous Active Control of Flexural and Extensional Power Flow in Beams," in Proceedings of International Congress on Recent Developments in Air and Structure-borne Sound and Vibration (Auburn University, Auburn, AL, 1990), pp. 657-662.

${ }^{10}$ C. F. Ross, "An Algorithm for Designing a Broadband Active Control System," J. Sound Vib. 80, 373-380 (1982).

${ }^{11}$ L. J. Erickson and M. C. Allie, "A Digital Sound Control System for Use in Turbulent Flows," Noise-Con 87, 365-370 (1987).

${ }^{12}$ L. J. Erikson, M. C. Allie, and R. A. Greiner, "The Selection and Application of an IIR Adaptive Filter for Use in Active Control Attenuation," IEEE Trans. Acoust. Speech Signal Process. ASSP-35, 433-437 (1987).

${ }^{13}$ J. V. Warner and R. J. Bernhard, "Digital Control of Sound Fields in Three-dimensional Enclosures," AIAA 11th Aeroacoustics Conference, 1-8 (1987).

${ }^{14}$ T. J. Sutton, S. J. Elliot, P. A. Nelson, and I. Moore, "Active Control of Multiple-source Random Sound in Enclosures," Proc. Inst. Acoust. 12, 689-693 (1990).

${ }^{15}$ S. J. Elliot, I. M. Stothers, and L. Billet, "Adaptive Feedforward Control of Flexural Waves Propagating in a Beam," Proc. Inst. Acoust. 12, 613-622 (1990).

${ }^{16}$ J. S. Vipperman, R. A. Burdisso, and C. R. Fuller, "Active Control of Broadband Structural Vibration Using the LMS Adaptive Algorithm," J. Sound Vib. (to be published) (1993).

${ }^{17}$ P. A. Nelson, J. K. Hammond, P. Joseph, and S. J. Elliott, "Active Control of Stationary Random Sound Fields," J. Acoust. Soc. Am. 87, 963-975 (1990).

${ }^{18}$ P. M. Joplin and P. A. Nelson, "Active Control of Low-frequency Random Sound in Enclosures,” J. Acoust. Soc. Am. 87, 2396-2404 (1990).

${ }^{19}$ B. Widrow and S. D. Stearns, Adaptive Signal Processing (PrenticeHall, New York, 1985).

${ }^{20}$ S. L. Marples, Jr., Digital Spectral Analysis with Applications (PrenticeHall, New York, 1987).

${ }^{21}$ R. Isermann, Digital Control Systems (Springer-Verlag, Berlin, 1981). 